\title{
Primary Adrenal Squamous Cell Carcinoma in a Single Kidney Patient: Case Report and Review of Literature
}

\section{Mehdi Kardoust Parizi}

Department of Urology, Shariati Hospital, Tehran University of Medical Sciences, Tehran, Iran

Received February 3, 2019; Accepted May 2, 2019.

Key words: Squamous cell carcinoma - Adrenal - Malignancy - Adrenalectomy

Abstract: The adrenal gland is a common metastatic site of squamous cell carcinoma (SCC), however primary adrenal SCC has not been reported in literature. In this case report, we presented the first case of primary adrenal SCC.

A 53-year-old man presented with chronic right flank pain. Abdominopelvic computed tomography (CT) confirmed left kidney agenesis and a soft tissue density mass measuring about $40 \times 30 \mathrm{~mm}$ in the right adrenal gland. Adrenal functional assessment including metanephrine, normetanephrine and vanillyl mandelic acid were normal. The patient underwent surgical resection of right adrenal mass. Pathology report revealed adrenal SCC. Immunohistochemistry evaluation demonstrated positive staining for P63, CK, CD10, CK7, chromogranin, neuron specific enolase, and negative staining for alpha-inhibin, neurofilament, CK20, and tyrosine hydroxylase that were compatible with SCC. All other assessments to find primary site of SCC including upper and lower gastro-intestinal endoscopy, chest CT scan, positron emission tomography scan, and bronchoscopy demonstrated normal findings. To our knowledge, this is the first case of primary adrenal SCC without any evidence of metastatic nature of such adrenal involvement by SCC.

Mailing Address: Mehdi Kardoust Parizi, MD., Department of Urology, Shariati Hospital, Tehran University of Medical Sciences, north karegar street, Tehran, Iran; Phone: +989 122570 482; e-mail: m.kardoust@yahoo.com 


\section{Introduction}

Squamous cell carcinoma (SCC) of the adrenal gland is usually diagnosed as a metastatic disease of another organ origin such as lung (Toogood et al., 2014). However, the primary adrenal SCC has not been reported in literature. The management of the local and metastatic SCC in adrenal gland could be different. Due to lack of data regarding the effective treatment for primary involvement of adrenal gland with SCC, we present this case report, a single kidney patient with primary adrenal SCC and no evidence of tumour origin in other organs to elucidate surgical local management of such rare tumour.

\section{Case report}

A 53-year-old man presented with dull and positional right flank pain for 3 months. Ultrasonography imaging revealed a $40 \times 30 \mathrm{~mm}$ hypoechoic lobulated mass at the anatomical site of right adrenal incidentally unrelated to the patient's symptoms. Left kidney was not found on ultrasonography in anatomical site. The patient had no history of left kidney surgery, crisis of hypertension, flashing and weight loss. Adrenal functional assessment included serum renin, potassium, cortisol and 24-hour urine evaluation (normetanephrine, metanephrine, and vanillyl mandelic acid) were in normal range. Metastatic workup, consisting of serum liver function tests, serum alkaline phosphatase and chest computed tomography (CT), revealed no abnormalities. Abdominopelvic CT confirmed a soft tissue density mass (38 Hounsfield unit) in pre-contrast imaging with mild enhancement $(63$ Hounsfield unit) measuring about $40 \times 30 \mathrm{~mm}$ arising from the right adrenal gland. Left kidney was not seen. However left adrenal was found in anatomical site (Figure 1).

Left ureteral orifice was not seen in cystoscopy. The patient underwent surgical excision of the right adrenal mass. Final pathology demonstrated highly malignant neoplastic tissue composed of sheets and nest of anaplastic cells. Individual cells were large with hyperchromatic nuclei. In some foci there were squamous differentiated and rare dyskeratotic cells (Figure 2). The invasive tumour cells invaded to the nearly all parts of adrenal gland with tiny remnant of normal adrenal tissue.
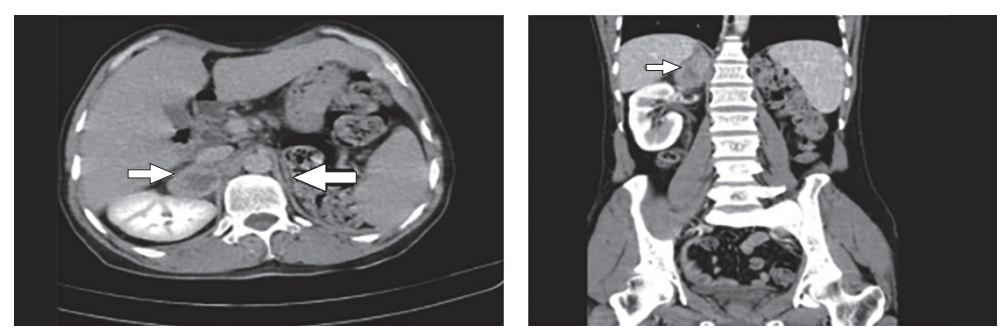

Figure 1 - Abdominopelvic computed tomography showing soft tissue mass measuring about $40 \times 30 \mathrm{~mm}$ above right kidney. Left kidney is absent (white and black arrows indicate right adrenal mass and left normal adrenal gland, respectively). 

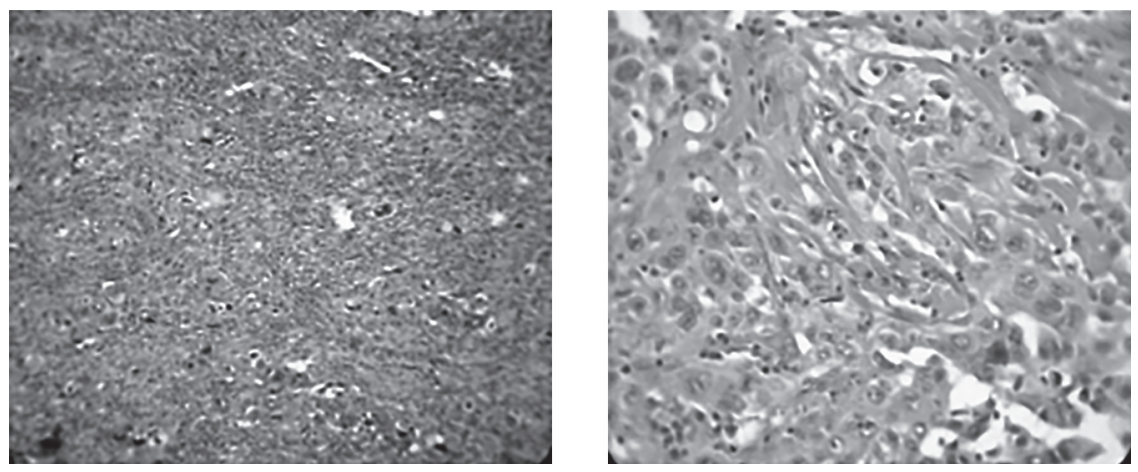

Figure 2 - High malignant tissue composed of sheets and nests of anaplastic cells, with hyperchromatic nuclei (haematoxylin and eosin stain).

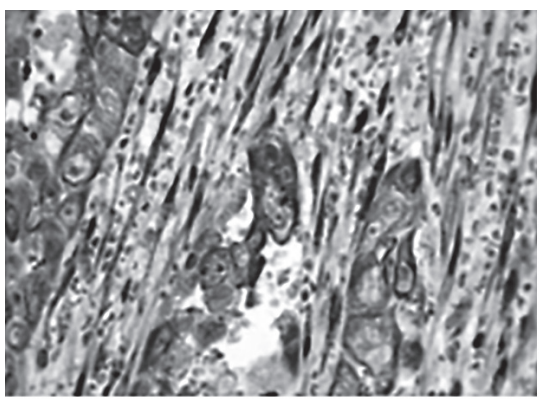

A

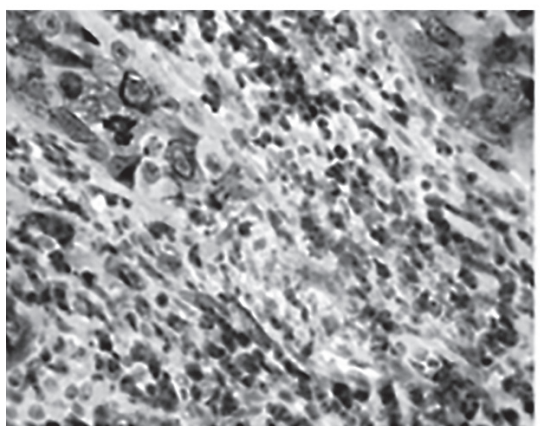

C

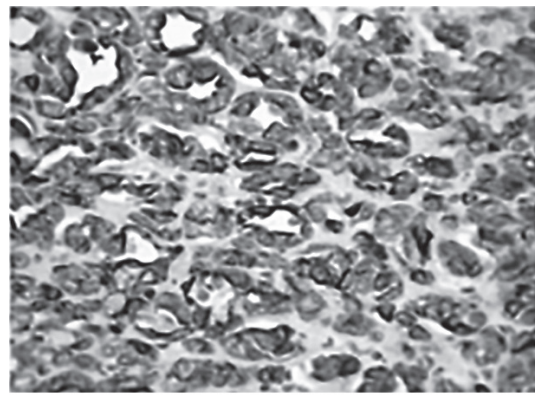

B

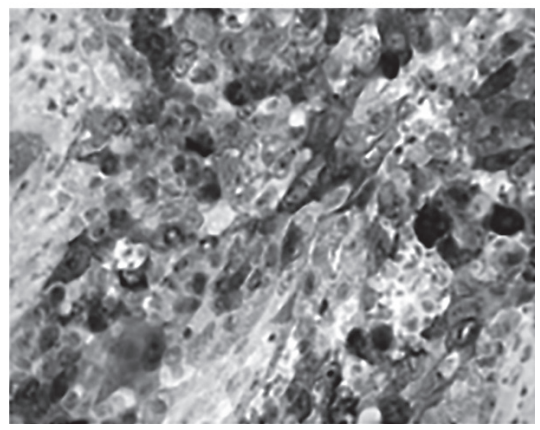

D

Figure 3 - Immunohistochemistry evaluation; positive staining for NSE (A), CK7 (B), CK10 (C), and calretinin (D).

Immunohistochemistry $(\mathrm{IHC})$ evaluation demonstrated the nature of the right adrenal mass - squamous cell carcinoma. In IHC assessment, we found positive staining for P63, CK, CD10, CK7, chromogranin, neuron specific enolase, and negative for alpha-inhibin, neurofilament, CK20, and tyrosine hydroxylase (Figure 3). 
All other evaluations included complete physical examination (especially skin with focus to find cutaneous origin of primary SCC), upper and lower gastrointestinal endoscopy, and bronchoscopy were normal and revealed no primary site of squamous cell carcinoma. Positron emission tomography scan demonstrated no other primary site for SCC. We did not find any signs and symptoms of adrenal deficiency after surgery as a result of contralateral normal adrenal function. Seven months after surgical resection of tumour, the patient was symptom free and follow-up assessment represented no local tumour recurrence or distant metastasis.

\section{Discussion}

The adrenal glands are composed of two embryologically distinct components with endocrine and neurocrine functions. The adrenal cortex originates from the coelomic mesoderm of the urogenital ridge and the medulla arises from neural crest tissue (Barwick et al., 2005).

The majority of adrenal masses are benign and asymptomatic; therefore these tumours usually are discovered accidentally in imaging or autopsy (Young, 2007). Most of the adrenal incidentalomas are diagnosed as adrenocortical adenoma. However, adrenocortical carcinoma, pheochromocytoma and metastatic carcinoma can be found in some cases with adrenal mass.

Adrenal neoplastic diseases originate from the adrenal cortex and medulla, or secondary from the other organs due to high vascularity of the adrenal glands. Primary adrenal malignancies include adrenocortical carcinoma, primary adrenal lymphoma and malignant pheochromocytomas. Lungs, breasts, kidneys, pancreas, and gastrointestinal tract are the most common malignancies with capability of metastasis to the adrenal glands (Toogood et al., 2014). Adrenal SCC is extremely rare, and it is always determined as a secondary tumour.

All types of lung cancer have the propensity for metastasis to the adrenal glands. Sometimes, the lung cancer is asymptomatic, and the first clinical manifestation is adrenal involvement. Therefore, lung CT scan should be the first diagnostic approach for adrenal masses with suspicious of a metastatic disease (Han et al., 2018). Lung cancerous cells spread hematogenous or lymphogenous to the adrenal glands. Therefore, the type of tumour diffusion affects histological pattern of the adrenal metastatic tumour. In lung cancer patients with early stage disease, the lymphogenous and ipsilateral adrenal involvement are more common and associate with good prognosis with adrenalectomy. Isolated ipsilateral adrenal metastasis should be considered as a regional disease with better oncological outcome after treatment (Kocijanc et al., 2003).

Besides lungs, in few cases, the other organs have been reported as an origin of metastatic adrenal SCC. In a case report, SCC of buccal mucosa was found as the source of adrenal metastasis. Distant metastasis is very rare in buccal SCC and most of the time it happens in lungs, bone, liver and skin (Manir et al., 2017). 
Although neural crest cells give rise to multiple cell types such as melanocytes (Noisa and Raivio, 2014), the direct association between this embryologic tissue and squamous cells has not been described. Coelomic epithelium as an embryonic origin of adrenal cortex contributes to development of organs involved by SCC such as lung, and gastrointestinal tract (Ariza et al., 2016). However, the role of this embryonic structure as a primary origin of adrenal SCC has not been demonstrated to date. SCC usually develops at surface tissues such as skin that originates from embryonic surface ectoderm. The common embryonic origin of the surface and neural ectoderm (the origin of the neural crest) may explain such primary rare adrenal tumour. Nevertheless, future genetic and histological assessments are needed to confirm this hypothesis.

Malignant transformation to SCC in other organs (such as ovaries) has been elucidated recently (Chiang et al., 2017), and could be used to describe development of SCC in unusual anatomical sites. Nevertheless, tumour transformation to SCC in adrenal gland has not been reported. This rarity could be due to the difference between cell origin of SCC and adrenal benign and malignant lesions. To the best of our knowledge, this case is the first case of primary adrenal SCC.

Surgical resection is an acceptable treatment for the primary adrenal tumours and isolated adrenal metastasis. Chemotherapy usually does not improve survival and sometimes, because of side effects, it could make patient's condition worse (Choi et al., 2011). Open, laparoscopic and robotic adrenal mass resection are acceptable surgical procedures for the management of adrenal masses (Agrusa et al., 2017). We performed open adrenalectomy by a thoracoabdominal incision. We considered no adjuvant therapy after the surgery. Seven months after surgery, our assessments including physical examination, laboratory tests and imaging modalities confirmed no evidence of disease relapse.

\section{Conclusion}

Adrenal SCC has been known as a secondary tumour. However, we present the first primary adrenal SCC treated with surgical resection. Future studies may bring more on pathophysiology, treatment modalities and follow-up methods to improve survival of patients with this extremely rare disease.

\section{References}

Agrusa, A., Romano, G., Navarra, G., Conzo, G., Pantuso, G., Buono, G. D., Citarrella, R., Galia, M., Monte, A. L., Cucinella, G., Gulotta, G. (2017) Innovation in endocrine surgery: Robotic versus laparoscopic adrenalectomy. Meta-analysis and systematic literature review. Oncotarget 8, 102392-102400.

Ariza, L., Carmona, R., Cañete, A., Cano, E., Muñoz-Chápuli, R. (2016) Coelomic epithelium-derived cells in visceral morphogenesis. Dev. Dyn. 245, 307-322.

Barwick, T. D., Malhotraa, A., Webba, J. A. W., Savageb, M. O., Reznek, R. H. (2005) Embryology of the adrenal glands and its relevance to diagnostic imaging. Clin. Radiol. 60, 953-959.

Chiang, A. J., Chen, M. Y., Weng, C. S., Lin, H., Lu, C. H., Wang, P. H., Huang, Y. F., Chiang, Y. C., Yu, M. H., Chang, C. L. (2017) Malignant transformation of ovarian mature cystic teratoma into squamous cell carcinoma: a Taiwanese Gynecologic Oncology Group (TGOG) study. J. Gynecol. Oncol. 28, e69. 
Choi, J. J., Buttrick, S., Zakashansky, K., Nezhat, F., Chin, E. H. (2011) Laparoscopic adrenalectomy for isolated adrenal metastasis from cervical squamous cell carcinoma and endometrial adenocarcinoma. Gynecol. Oncol. 122, 684-685.

Han, H., Qiao, P., Jiang, X., Wang, B., Zhang, X. (2018) Acute flank abdominal pain as the chief complaint of spontaneous adrenal hemorrhage secondary to metastatic lung cancer. Urol. Case Rep. 16, 65-68.

Kocijanc, I., Vidmar, K., Zwitter, M., Snoj, M. (2003) The significance of adrenal metastases from lung carcinoma. Eur. J. Surg. Oncol. 29, 87-88.

Manir, K. S., Chakraborty, S., Biswas, S., Mondal, D., Paul, S., Roy, C. (2017) An unusual adrenal gland metastasis in a case of metastasis cell carcinoma of buccal mucosa. Int. J. Med. Pharm. Case Reports 9, 1-5.

Noisa, P., Raivio, T. (2014) Neural crest cells: From developmental biology to clinical interventions. Birth Defects Res. C Embryo Today 102, 263-274.

Toogood, V., Milliken, S., Morey, A., Samaras, K. (2014) Adrenal tumors: How to establish malignancy. BMJ Case Rep. 2014, bcr2014203736.

Young, W. (2007) The incidentally discovered adrenal mass. N. Engl. J. Med. 356, 601-610. 